EPJ Web of Conferences 66, 10006 (2014)

DOI: 10.1051/epjconf/ 20146610006

(C) Owned by the authors, published by EDP Sciences, 2014

\title{
CHIPS_TPT models for exclusive Geant4 simulation of neutron-nuclear reactions at low energies
}

\author{
Mikhail V. Kosov ${ }^{1,2,3 a}$, Ilya V. Kudinov ${ }^{1}$, and Dmitry I. Savin ${ }^{1,3}$ \\ ${ }^{1}$ All-Russia Research Institute of Automatics (VNIIA), Moscow, Russia \\ ${ }^{2}$ Institute for Theoretical and Experimental Physics, Moscow, Russia \\ ${ }^{3}$ Moscow Institute of Physics and Technology, Moscow, Russia
}

\begin{abstract}
A novel TPT code (Toolkit for Particle Transport), which is included in CHIPS_TPT physics list for Geant4 simulations, is briefly overviewed. Underlying concept of exclusive modelling is introduced and its beneficial features are illustrated with several examples. Widely used neutron Monte Carlo codes, MCNP and Geant4/HP, are based on inclusive algorithms that independently model neutron state change and secondary particles production while tracking. The exclusive approach implemented in TPT overcomes this unphysical separation and makes it possible to allow for kinematic restrictions as well as correlated emission of gamma-rays and secondaries.
\end{abstract}

\section{Introduction}

The Physics list CHIPS_TPT for Geant4 [1] applications combines a universal package CHIPS (CHiral Invariant Phase Space), which simulates high-energy hadronic interactions [2], with a newlydeveloped package TPT (Toolkit for Particle Transport) dedicated to exclusive modeling of neutronnuclear reactions below $20 \mathrm{MeV}$ [3]. Due to tight kinematic restrictions at low energies, quark-level algorithms implemented in CHIPS become inefficient and should be supplemented by other models. In Geant4 libraries low-energy neutron physics is represented by the HP (High Precision) package which follows an inclusive approach. TPT provides a novel alternative to HP, as it possesses several beneficial features that are briefly reviewed hereafter.

TPT is being developed in All-Russia Research Institute of Automatics (VNIIA) since 2012. The code is programmed in $\mathrm{C}++$ in accordance with Object-Oriented Programming paradigm. Freely distributable class libraries Geant 4 are used in TPT as a basic infrastructure to perform simulations. The principal TPT concept, distinguishing it from other neutron Monte Carlo codes (e.g. MCNP [4], HP), is the exclusive approach which utilizes exclusive cross sections to specify reaction products and excitation level of the daughter nucleus which is further de-excited by sequential gamma emission. Due to such an approach TPT strictly conserves energy and momentum at each algorithm step and, as a result, in every single interaction. In contrast, the inclusive codes reproduce energy conservation, as well as secondary particles' yields, only as mean values for numerous simulated events.

The exclusive TPT algorithms require a special nuclear data base, which was constructed by collecting and reinterpreting data from TALYS 1.4 [5] and ENSDF [6] (nuclear levels, branching ratios), and ENDF/B-VII.1 [7] (excitation functions). Additionally, for isotopes missing in ENDF/B-

\footnotetext{
${ }^{\text {a }}$ Corresponding author: kosov@vniia.ru
} 
VII.1 cross sections from TENDL-2011 [8] were utilized ( migration to TENDL-2012 has been performed). At present, TPT functionality covers inelastic scattering, binary reactions: (n,p), (n,d), $(\mathrm{n}, \mathrm{t}),\left(\mathrm{n},{ }^{3} \mathrm{He}\right),(\mathrm{n}, \mathrm{a})$; triple reactions $(\mathrm{n}, \mathrm{np}),(\mathrm{n}, \mathrm{nd}),(\mathrm{n}, \mathrm{nt}),\left(\mathrm{n}, \mathrm{n}^{3} \mathrm{He}\right),(\mathrm{n}, \mathrm{na})$ and few other. Elastic scattering and radiative capture are under development (currently these processes are taken from HP). Fission, reverse binary reactions like $(p, n)$ and neutron multiplication reactions like $(n, 3 n)$ are planned to be implemented in the future.

\section{Phenomena reproduced by only exclusive modelling}

The exclusive modeling enables TPT to reproduce phenomena that are beyond the inclusive modeling capabilities. One of those is correlated emission of gammas and other secondary particles (Fig. 1). In contrast, MCNP never produces multiple gamma rays in a single interaction, as explicitly demonstrated in [9].

Another example is kinematic effects such as gamma line broadening due to the motion of the excited nucleus. Let us consider the ${ }^{16} \mathrm{O}(\mathrm{n}, \mathrm{a}){ }^{13} \mathrm{C}$ reaction and the corresponding photon production (Figs. 2-3). Level 3.853 of ${ }^{13} \mathrm{C}$ is relatively long-lived (8.6 ps) so that the excited nucleus has time to stop before the gamma emission, thus providing a narrow 3.853 line. Level 3.684 has a relatively short lifetime $(1.1 \mathrm{fs})$, which leads to a Doppler-broadened gamma-line in the case of direct excitation of this level (100\% decay in ground state). In the case of indirect excitation, i.e. transition from 3.853 to 3.684 (BR 16.9\%), the gamma quanta have a low energy dispersion, similar to that of 3.853 line. Comparison of numerical and experimental data (Fig. 2) indicates over-broadening of the simulated lines. This discrepancy has a clear source: in TPT nucleus stopping during the excitation lifetime is not taken into account for the moment. However, it should be highlighted that the inclusive MC codes (MCNP, HP) cannot reproduce the phenomenon of Doppler broadening in principle: only fixedenergy gammas based on tabulated values are emitted.

Also, strict energy conservation as realized in TPT is essential for problems related to neutron KERMA in gas media.

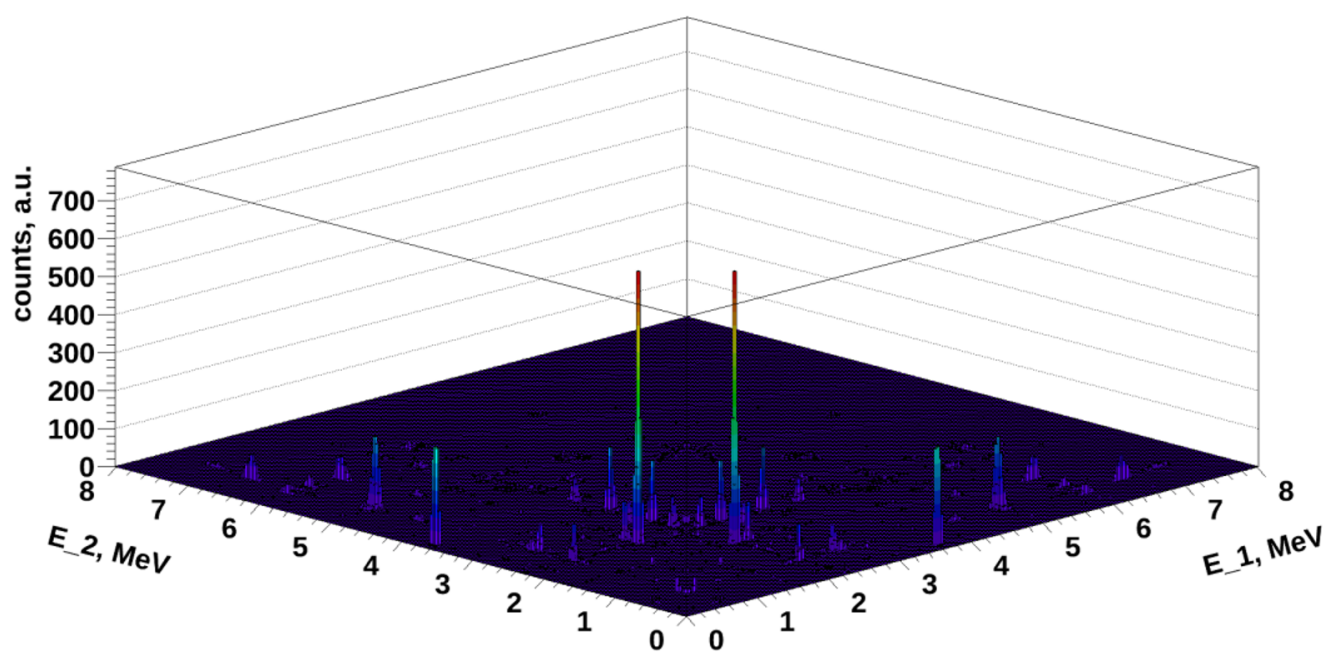

Figure 1. Simulated 2D gamma correlation spectrum of ${ }^{14} \mathrm{~N}$ irradiated by $14 \mathrm{MeV}$ neutrons (horizontal axes correspond to energies of the first and the second gammas). Numerical results obtained with TPT are presented. The histogram is symmetrical due to construction procedure. 


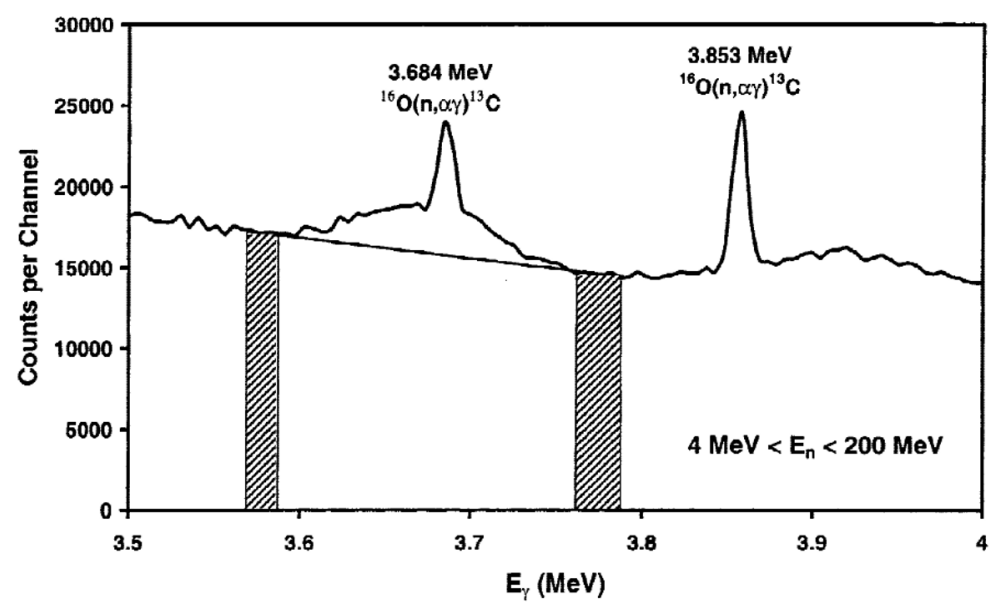

Figure 2. Experimentally measured gamma spectrum of oxygen in the vicinity of 3.684 and $3.853 \mathrm{MeV}$ lines produced by ${ }^{16} \mathrm{O}(\mathrm{n}, \mathrm{a}){ }^{13} \mathrm{C}$ reaction. Reprinted from [10].

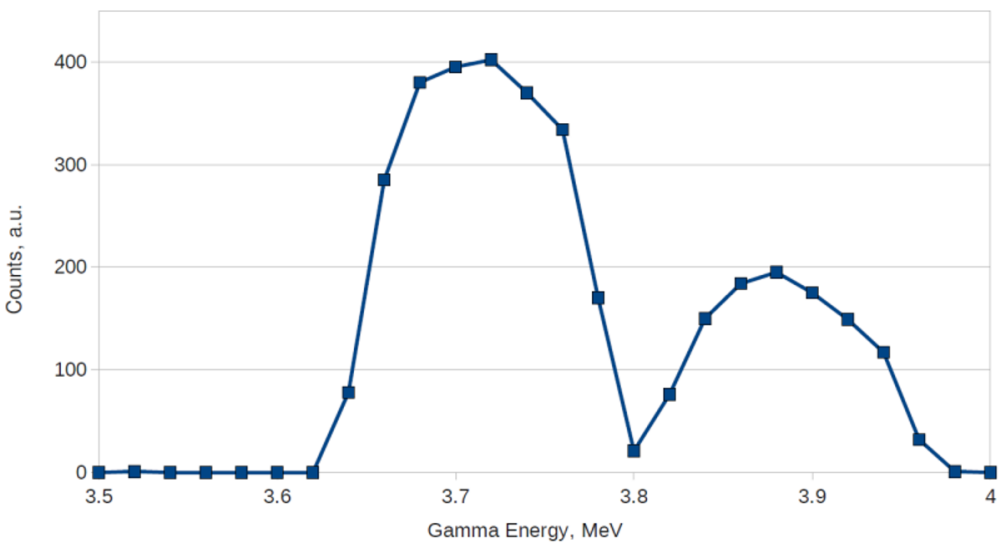

Figure 3. Gamma spectrum of oxygen in the vicinity of 3.684 and $3.853 \mathrm{MeV}$ lines produced by ${ }^{16} \mathrm{O}(\mathrm{n}, \mathrm{a}){ }^{13} \mathrm{C}$ reaction. Numerical results obtained with TPT are presented (squares).

\section{TPT accuracy in 1D traditional gamma spectra reconstruction}

In problems that could be treated both by the inclusive codes and by TPT, the latter provides accuracy comparable to MCNP and surpasses HP. To illustrate this in Fig. 4 gamma spectra simulated by TPT and HP are compared with the measured one for a melamine sample (chemical formula $\mathrm{C}_{3} \mathrm{H}_{6} \mathrm{~N}_{6}$, density $0.83 \mathrm{~g} / \mathrm{cm}^{3}$, sample size $8 \times 8 \times 16 \mathrm{~cm}^{3}$ ). The measurement was performed in VNIIA utilizing tagged neutrons technique. A commercial VNIIA d-t generator with a built-in matrix alpha-detector was used as a neutron source. Induced gamma rays were detected by a cylindrical BGO scintillator (diameter $6.35 \mathrm{~cm}$, height $6.35 \mathrm{~cm}$, aluminum casing). Due to the rough calibration procedure, a slight shift of the recorded spectrum with respect to the energy axis is observed in Fig. 4. Therefore experimental values should be regarded as preliminary.

The plots indicate that TPT provides better agreement with the measured data compared to HP. The latter overestimates the signal at high energies and strongly overrates the intensity of the 1.635 and $2.313 \mathrm{MeV}$ lines from inelastic scattering on ${ }^{14} \mathrm{~N}$. In addition, if flag «G4NEUTRONHP_DO_NOT_ADJUST_FINAL_STATE» is switched off, the HP algorithm 
attempts to enforce energy conservation by emitting fictitious gammas that lead to the non-physical line near $1 \mathrm{MeV}$.
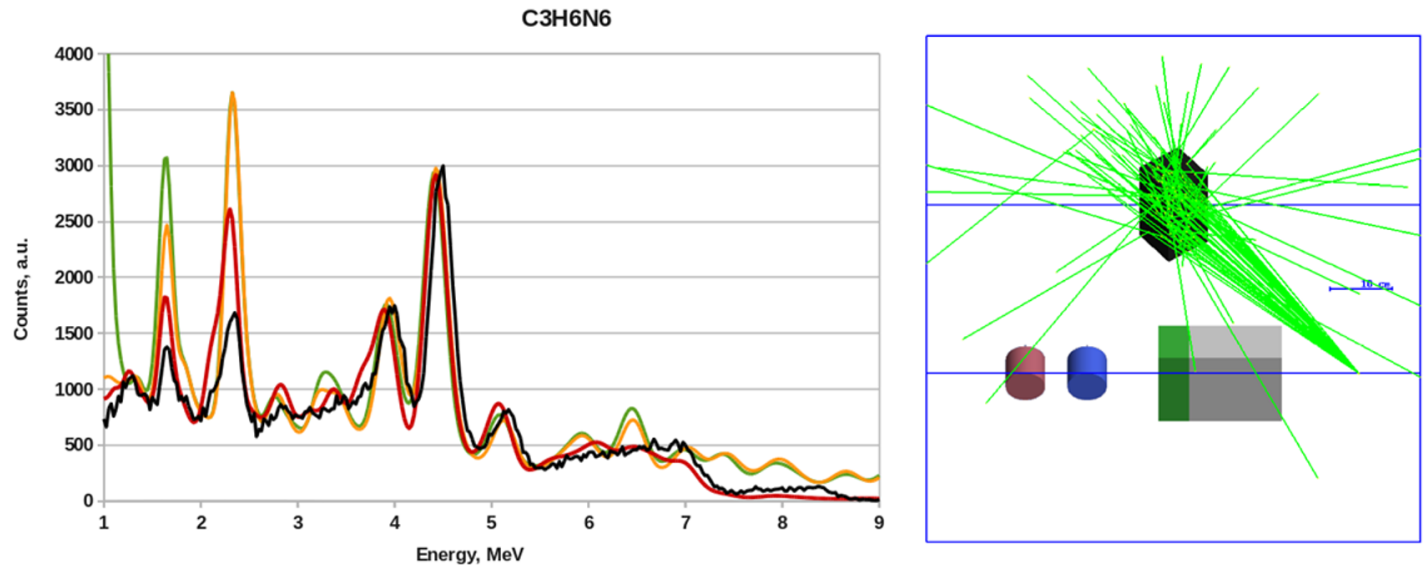

Figure 4. Left: Melamine gamma spectrum induced by $14 \mathrm{MeV}$ neutrons: measured (black), simulated by TPT (red), by HP with flags recommended (orange), by HP without flags (green). Right: scheme of the experiment: tagged neutrons and gamma tracks (light green), melamine sample (black), BGO scintillator (blue), LYSO scintillator (pink), tungsten shield (grey), lead shield (green).

\section{Conclusions}

TPT is a next-generation Monte Carlo code for neutron interactions modeling below $20 \mathrm{MeV}$. TPT is based upon the treatment of exclusive nuclear reactions which enables strict energy and momentum conservation in every collision. As opposed to the widely spread inclusive codes (MCNP, Geant4/HP), TPT can reproduce the correlated emission of gammas and other secondary particles, as well as kinematical effects related to the excited nucleus motion. TPT is under development now and its functionality will expand to cover several reactions not implemented at the moment. However, at present the TPT accuracy of prompt gamma spectrum modeling (i.e. without slowing-down and radiative capture) is not inferior to MCNP's accuracy and exceeds that of Geant4/HP. Practical applications that may benefit from TPT-based simulations include -but are not limited to- neutron technologies for oil/gas well logging, explosives detection in cargo and luggage, feed composition control at cement plants.

\section{References}

1. http://geant4.cern.ch/

2. M.V. Kossov, Manual for the CHIPS event generator, KEK 2000-17 (2001)

3. http://vniia.ru/cfpi/eng/wptpt.html

4. http://mcnp.lanl.gov/

5. http://www.talys.eu

6. http://www.nndc.bnl.gov/ensdf/

7. http://www.nndc.bnl.gov/endf/b7.1/

8. ftp://ttp.nrg.eu/pub/www/talys/tendl2011/tendl2011.html

9. T. Wilcox et al., Progr. Nucl. Energy, 63, 1 (2013)

10. R.O. Nelson et al., Nucl. Sci. Eng. 138, 105 (2001) 